Instituto Internacional de Investigación y Desarrollo Tecnológico Educativo INDTEC, C.A.

DOI: https://doi.org/10.29394/scientific.issn.2542-2987.2017.2.5.10.182-200

OAI-PMH: http://www.indteca.com/ojs/index.php/Revista Scientific/oai

\title{
Conservación del Ambiente Basado en la Construcción de Cercas Vivas
}

\author{
Autora: Nancy Yuraima Veras Burgos \\ Universidad Pedagógica Experimental Libertador, UPEL \\ nancyura@hotmail.es \\ Barinas, Venezuela
}

\section{Resumen}

La presente investigación reflexiona sobre la conservación del ambiente, considerando, la necesidad de preservar los recursos naturales de una manera cónsona con el desarrollo de la educación ambiental, en tal sentido plantea como meta fundamental desarrollar un plan para la conservación del ambiente basado en la construcción de cercas vivas en la escuela básica pablo María Alisa Barinas estado Barinas, para los aportes teóricos que sustentan la investigación se consultaron, diferentes autores y enfoque relativos a la conservación del medio ambiente. La misma se desarrollará dentro del paradigma cualitativo y centrado en una investigación acción participante, en relación al diseño es de campo, por cuanto la recolección de datos se realizó directamente de los sujetos investigados, en lo que respecta a los informantes, se tomaron, cuatros (04) sujetos objetos de estudio, en cuanto a la técnica de recolección de datos se utilizó la entrevista y la observación. Finalmente, los resultados formarán la propuesta de aplicación para contribuir en la construcción de las cercas vivas.

Palabras clave: plan; preservación: ambiente; construcción; cercas; vivas. 


\section{Conservation of Environment Based on Fence Construction Alive}

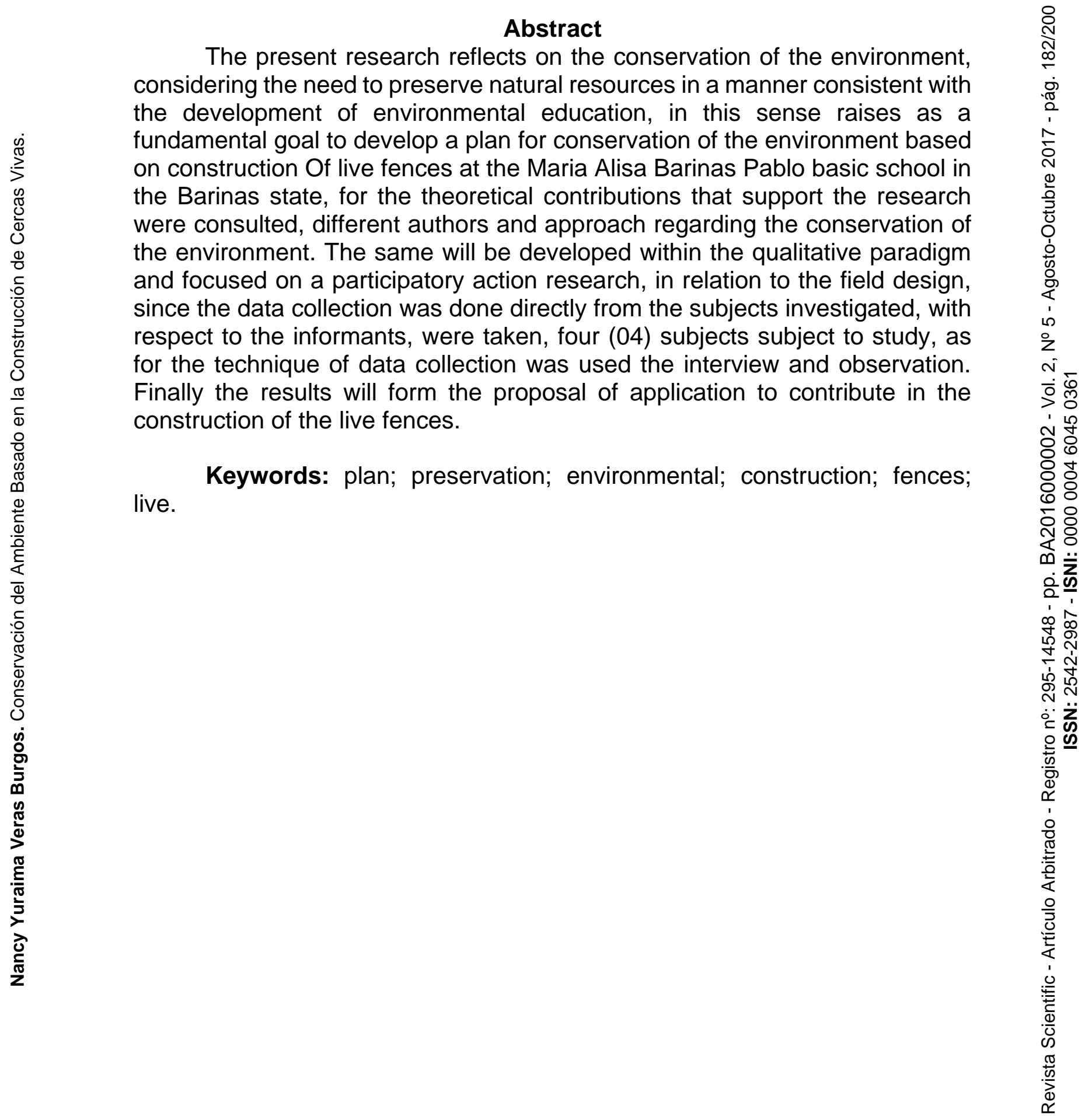

Date Received: 06-02-2017

Date Acceptance: 16-03-2017 


\section{Introducción}

La conservación del ambiente es tema de interés por cuanto el deterioro ha causado grandes impactos en los ecosistemas, al respecto las Naciones Unidas ONU (2014) expresaron que, la crisis ecológica a nivel mundial básicamente está caracterizada por el aumento exponencial acumulativo y sinérgico de las presiones a las que se ve sometido el delicado equilibrio ecológico, el cual transfiere al presente histórico peligros en los que se advierte la posibilidad de provocar una ruptura ambiental sin precedente, ya sea en relación a los aumentos constitutivos básicos del medio natural (Tierra, agua y aire), a las modificaciones de las sucesiones esenciales para las dinámicas biológicas y sociales o a los aspectos que se vinculan a la explotación de los recursos, o a los desechos que se ocasionan la perdida de la biodiversidad, así como la perdida de los bosques, los cuales son el pulmón vegetal que da vida al planeta.

Continúa expresando la fuente, que los gobiernos a nivel mundial, deben tomar en serio esta problemática, para evitar un desastre a nivel ambiental, implementando políticas que permitan el desarrollo de una educación ambiental, en beneficio del planeta.

De igual manera en Latino América, Yepes (2012) expreso que, los problemas ambientales, han superado los niveles, Se calcula que en los últimos 30 años se han talado en América Latina cerca de 2 millones de kilómetros cuadrados de bosques, es decir, una superficie superior a la del territorio mexicano, al ser superior a los $50 \mathrm{mil} \mathrm{km2} \mathrm{por} \mathrm{año} \mathrm{la} \mathrm{tasa} \mathrm{actual} \mathrm{de}$ deforestación es "particularmente grave", el hecho de que la población más expuesta a los impactos del cambio global en la región de América Latina y Caribe es la que se asienta en la zona caribeña, en particular la que habita en pequeños estados insulares.

De igual manera expresa la fuente que En Argentina un dato ilustra mejor que nada la magnitud del problema: en los últimos cinco años, la tala 
arrasó con 1,3 millones de hectáreas en el país, según la Unidad de Monitoreo del Sistema de Evaluación Forestal de la Dirección de Bosques, a partir de la lectura de fotos satelitales que aporta la Comisión Nacional de Actividades Espaciales, lo que provoca pérdida de diversidad biológica a nivel genético, poblacional y eco sistémico.

En este sentido, Autores como Moncada (2014) y Novo (1996) citados por Mujica, (2014) "plantean que la preservación del ambiente Parte de explicar, orientar, organizar y proyectar la educación ambiental en las instituciones educativas y las comunidades, son algunas de las tareas de mayor importancia en la ciencia de la pedagogía". (pág. 58).

El Autor citado señala, que la educación ambiental es el motor principal para la preservación del ambiente a través de los mecanismos de acción que se puedan aplicar, tanto en los centros educativos como en el entorno comunitario, visto la necesidad preponderante de recuperar la naturaleza verde a través de la aplicación de planes que permitan aumentar el reservorio arbóreo pulmón de nuestro planeta, incrementando el cultivo de cercas vivas en áreas verdes, en beneficio de la preservación del medio ambiente siendo que estas son una opción real de conservación de los recursos naturales, en especial de la biodiversidad, en sistemas de producción de tipo sostenible.

En el caso de Venezuela, según La Ramírez (2011), expresa que no está al margen de la realidad representada a nivel mundial en cuanto al incremento del resquebrajamiento o deterioro ambiental, sus causas o motivos son las indicadas con anterioridad, sumándose además en el aspecto económico, la explotación minero petrolera. Por otra parte, también en ecosistemas de gran fragilidad como las sabanas y selvas vírgenes, son destruidas para los desarrollos agrícolas, ganaderos y forestales.

A todos estos planteamientos este informe plantea que Resulta necesario fortalecer los procesos de vigilancia, reforzar la gestión en áreas naturales protegidas y educar a la población, de igual manera se debe hacer 
cumplir la legislación vigente, fortalecer los procesos de vigilancia y control y hacer programas de educación y comunicación dirigidos a todos los actores involucrados, así mismo consideró que es necesario fortalecer la capacidad del Ministerio del Poder Popular para el Ambiente para hacer una eficaz supervisión y control de las actividades, reforzar los programas de control de contingencias, y capacitar al personal en temas de valoración y gestión ambiental.

Cabe señalar según el Banco Mundial (2012), que uno de los desafíos que presenta Venezuela, es la práctica de una educación ambiental que permita concientizar, a la población sobre la necesidad de defender el medio ambiente, y los recursos naturales, siendo que estos han sido utilizados de manera adecuada, trayendo como resultado problemas ambientales, manifestándose estos en algunas regiones y zonas de Venezuela.

En los postulados de Maldonado (2012) expresa, que el deterioro ecológico de Venezuela no solo tiene su origen en el crecimiento económico sino también se manifiestan en la marginalidad social, lo que hace que la situación sea aún más compleja. Esta situación es el resultado de una ocupación irracional de los espacios que son reservorios naturales y consecuentemente del mal uso de estos reservorios verdes, que han venido utilizándose en proporción cada vez mayor, además la forma de utilizar estos recursos en el país, ha traído como consecuencia danos irreversible al ecosistema, entre ellos la perdida de los reservorios verdes de la nación, la contaminación ambiental entre otros problemas generado por la acción del hombre.

También refiere el autor que, la concepción de un mundo verde ha ganado espacios cada vez más importantes en nuestra sociedad, la necesidad de preservación del medio ambiente surge del hecho de percatarnos del grave daño que se ha ocasionado a los ecosistemas a través de la galopante transformación negativa que estos han sufrido debido a los intentos frustrados 
de desarrollo. No han comprendido la necesidad de tener un crecimiento económico que vaya de la mano con la preservación del ambiente, porque la depredación de la naturaleza para obtener beneficios económicos abundantes ha resultado más fácil que reponer los daños que se le originan al tomar sus recursos de manera indiscriminada.

De igual manera Vitales (2013) expreso que Venezuela, requiere incrementar estrategias para frenar el grave deterioro ambiental en sus reservas arbóreas siendo que estos pulmones vegetales son vitales para la sobrevivencias, considero que en años anteriores ha sido afectada por graves cambios climáticos, ocasionados por el mal uso de sus reservas, de igual manera manifiesto que se debe implementar mecanismos que permitan concientizar, sobre la inminente necesidad de incrementar la biodiversidad de la flora, por consiguiente insto a los organismos encargados, a realizar campañas ambientalistas que promuevan la producción de espacios verdes en las comunidades, a través de recolección de semillas y cultivos de árboles.

En lo relativo, a lo planteado con relación a la conservación del ambiente en el estado Barinas se evidencia por parte de la autora del estudio, cómo se alteran negativamente los espacios naturales, permitiéndose incluso la desaparición de grandes extensiones de recursos vegetales por la invasión indiscriminada de grupos humanos en sus áreas, los cuales se procuran un espacio para vivir.

Al respecto se observa que el gobierno nacional ha elevado dicha iniciativa, justificándola en muchas oportunidades, acarreando esto graves consecuencias para nuestros pulmones naturales vegetales. Dicha conducta ha ocasionado la deforestación de grandes extensiones de terrenos donde existe plantaciones de especies arbóreas, de esta manera, estos espacios son utilizados para la construcción de viviendas, sufriendo en ocasiones los embates de los elementos naturales como la lluvia, que originan la pérdida de 
bienes y vidas humanas, debido a la utilización inadecuada de zonas no aptos para la ubicación de viviendas.

De esta manera estos espacios al ser abandonados, por orden de las autoridades, dejan una estela de destrucción en la naturaleza, sin que existan políticas cónsonas que permitan su recuperación. Partiendo de la necesidad de conservar el medio ambiente, se crea la propuesta de construir cercas vivas siendo que esta es una propuesta ecológica considerando que las cercas vivas tienen una vital importancia, para la conservación de la biodiversidad en paisajes fragmentados o áreas productivas puesto que, los elementos arbóreos que éstas poseen, crean una variedad de hábitats que no están presentes en lugares manejados intensivamente por el hombre.

En lo que refiere a las cercas vivas Casasola (2009). Citado por Tobar (2010), "expreso las cercas vivas son hábitats perturbadas y manejadas por el hombre que han demostrado tener un papel importante en la conservación de los suelos, clima, agua y diversidad biológica”. (pág. 448).

Tomando en consideración, estas fundamentaciones, y en la búsqueda de mejorar el impacto ambiental, así como de educar bajo la práctica ambientalista, visto que existe la necesidad en la escuela Básica Pablo María Aliza, Además de carecer de cerca perimetral para el resguardo de su ambiente, así como de brindar a la comunidad estudiantil una educación ambiental de calidad, donde tanto los estudiantes como la comunidad educativa en general se beneficien, y puedan mejorar su calidad de vida en el medio rural .

A tal efecto con esta investigación se persigue, la construcción de cercas vivas para los alrededores de la escuela, de esta manera dar a conocer la importancia de la ecología, así como la conservación de los recursos naturales como son las especies arbóreas de nuestra región, con el serio compromiso de evitar la degradación del ambiente y la destrucción del entorno. 


\subsubsection{Propósito de la Investigación}

\subsubsection{Propósito General}

Desarrollar un plan para la conservación del ambiente basado en la construcción de cercas vivas en la escuela básica pablo María Alisa Barinas estado Barinas.

\section{Referentes Teóricos}

\subsection{Ambiente en el escenario de la Educación Ambiental}

Valero (2007), expreso que las concepciones de "ambiente" y "problemática ambiental" son básicas para emprender los procesos educativos ambientales. Al respecto varios autores han analizado las dimensiones ecológicas, sociales, culturales y económicas del concepto de ambiente; cada uno le atribuye cualidades específicas que se traducen en caracterizar de una forma compleja y global las interrelaciones de los elementos que conforman el sistema, donde el ser humano forma parte integrante de éste y sus acciones influyen y determinan el equilibrio del mismo.

De igual manera refleja la autora que, la complejidad de los sistemas es el escenario de análisis para la construcción de saberes desde la educación ambiental, cuya acción está orientada a analizar la problemática del hombrenaturaleza, sus relaciones directas por el uso de los recursos naturales como fuentes de materia prima y los impactos generados por el uso ilimitado de estos, asociados a ello, la pérdida de biodiversidad, la ocupación desordenada del territorio, la generación de fuentes contaminantes por efluentes y emisiones de los diversos procesos industriales, entre otros; posicionando así, el escenario de discusión desde los espacios locales, más próximos a los actores y su proyección con los espacios regionales, nacionales y globales. 


\subsection{Cercas vivas para la conservación del ambiente}

La FAO (2003), ha estimado que el $40 \%$ de la totalidad del territorio Centroamericano está constituido por tierras utilizadas para pastoreo. A su vez, un $60 \%$ de estas áreas de pasturas se encuentran degradadas, como consecuencia de la producción ganadera tradicional basada en el manejo de pasturas sin árboles. Esto ha ocasionado diferentes problemas ambientales, como la erosión y pérdida de la fertilización natural de los suelos, desertificación, pérdida de diversidad biológica, emisión de gases de efecto invernadero y contaminación de aguas. Asimismo, ha influido en el cambio de la composición de las especies de las comunidades bióticas, alteración en la función de los ecosistemas, propiciando la dispersión de especies exóticas y las que se benefician de la fragmentación del hábitat.

En tal sentido continua expresando la fuente que las cercas vivas y los árboles dispersos en potreros pueden contribuir a la conservación de la biodiversidad, debido a que pueden servir como corredores biológicos para la fauna y flora silvestre, las cercas vivas son elementos lineales que dependiendo de la estructura vertical o el número de estratos pueden generar hábitats, dependiendo de la abundancia y composición florística, estructura arbórea y arreglo espacial de las cercas en el agro paisaje, resultan de gran importancia para la conservación. Es decir que presentan una mayor complejidad estructural y mayor diversidad florística, pueden ayudar a conservar un mayor número de especies de fauna silvestres que las cercas vivas simples.

\section{Marco Metodológico}

\subsection{Naturaleza de la Investigación}

La presente investigación sobre la Educación Ambiental, se enmarca en un plan para la construcción de cercas vivas en la escuela básica Pablo María Alisa parroquia San Silvestre Municipio Barinas estado Barinas. La 
fundamentación epistemológica del estudio, se centra en el paradigma cualitativo e interpretativo, cuyos fundamentos teóricos lo constituye la fenomenología con la finalidad de comprender e interpretar la realidad. Martínez (2006), la fenomenología tiene las siguientes características:

(a) Es una investigación que trata de descubrir las estructuras esenciales de la conciencia (b) su fin es describir un fenómeno y descubrir en él la esencia (el éidos) válida universalmente, y útil científicamente (c) no se limita al estudio de casos (d) parte del estudio de casos, pues su meta consiste en alcanzar los principios generales a través de la intuición de la esencia (pág. 85).

Este método permite a los fenómenos manifestarse tal como son, describiendo las esencias de la conciencia pura, no desea excluir de su objeto de estudio nada de lo que se presenta a la conciencia; sin embargo, por otro lado, desea aceptar sólo lo que se presenta y, precisamente así como se presenta. Su fin no es tanto describir un fenómeno singular cuanto descubrir en él la esencia válida universalmente, y útil científicamente. Puesto que la conducta humana, lo que la gente dice y hace deriva de la forma en que define su mundo; la tarea del fenomenólogo es aprehender este proceso de interpretación, intentando ver las cosas desde el punto de vista de otras personas.

Las concepciones que sustentan el paradigma cualitativo e interpretativo sobre la educación ambiental en la preservación del medio ambiente, siguiendo a Habermas (1994), quien expreso que el paradigma cualitativo, interpretativo, tiene un marcado carácter humanista; le interesa el nivel racional (subjetivo) del conocimiento; pretende captar las relaciones internas, profundas, la intencionalidad de las acciones y percepciones de los sujetos, confiere más importancia a la comprensión, a la intencionalidad, que a la causalidad y la predicción. Asume el enfoque de una realidad modelada y 
construida, donde el investigador forma parte de esa realidad; entre el sujeto de la investigación y el objeto se establece una relación de interdependencia e interacción, enfatiza sobre la naturaleza interna del sujeto. Partiendo de esta concepción la finalidad de esta investigación es Diseñar un plan para la preservación del ambiente basado en la construcción de cercas vivas, en la búsqueda de conservar el medio ambiente, creando un ambiente de integración comunitaria.

\subsection{Tipo de Investigación}

En lo que refiere al tipo de investigación, se centra en la investigación acción participante, que de acuerdo Kurt (1944). La define como el estudio de una situación social para tratar de mejorar la calidad de la acción en la misma. Su objetivo consiste en proporcionar elementos de juicio crítico para la acción, y la validez de las teorías e hipótesis que genera depende más de su utilidad para ayudar a las personas a actuar de un modo más inteligente que de "pruebas científicas de verdad".

\subsection{Diseño de la Investigación}

El estudio quedo Afianzado dentro de un diseño de campo. En tal sentido Según el autor Arias (2012), define: La investigación de campo es aquella que consiste en la recolección de datos directamente de los sujetos investigados, o de la realidad donde ocurren los hechos (datos primarios), sin manipular o controlar variable alguna, es decir, el investigador obtiene la información, pero no altera las condiciones existentes. De allí su carácter de investigación no experimental.

Es importante reflejar que, por medio de la instrumentación del diseño establecido, se considera oportuno llevar a cabo un diagnóstico de la realidad que tiene que ver con el desarrollo de un plan para la conservación del ambiente basado en la construcción de cercas vivas en la escuela básica pablo 
María Alisa Parroquia San Silvestre Municipio Barinas estado Barinas

\subsection{Etapas Del Estudio.}

\subsubsection{Etapa Previa}

Clarificación de los presupuestos: Identificación de valores, creencias e intereses, conjeturas e hipótesis; para precisar su influencia en la investigación

\subsubsection{Etapa Descriptiva}

Se describe el fenómeno reflejando la realidad vivida por los sujetos, actuación, vivencias, situación, responsabilidad. Esta etapa se desarrolla en tres pasos:

1. Para esta investigación se seleccionaron las siguientes técnicas de recolección de información: Análisis documental y la entrevista.

2. Procedimientos aplicados para recabar la información.

3. Descripción protocolar: Reflejar el fenómeno tal como fue descrito por los entrevistados, ser lo más imparcial posible, evitar las ideas o prejuicios y recoger la información en su contexto natural.

\subsubsection{Etapa Estructural}

Estudiar detalladamente las descripciones previamente realizadas:

1. Delimitar áreas temáticas.

2. Determinar el tema central de cada área temática.

3. Expresar el tema central en lenguaje científico.

4. Integrar los temas centrales en estructuras descriptivas.

5. Integrar las estructuras particulares en una general.

6. Procesamiento final la de información. 


\subsection{Informantes}

La muestra en este estudio fue intencionada y quedo constituida por los actores sociales y/o informantes claves, que poseen el conocimiento status y tengan acceso a información inaccesible para el investigador, en correspondencia con Taylor y Bogdan (1998), quienes definen a los informantes claves como tipo de sujetos que son elegidos basados en fines que persigue una determinada investigación.

En el estudio, para la selección de los actores sociales se aplicó los criterios establecidos por el investigador, los mismos correspondió a dos (02) representantes y dos (02) estudiantes.

\subsection{Técnicas de investigación y recolección de datos}

En opinión de Martínez, M. (2008), la técnica indica "cómo se va a recoger la información y el instrumento señala cual información seleccionar" (pág. 428). De ahí que las técnicas a utilizar serán la entrevista.

La investigación cualitativa usa múltiples y útiles técnicas, para la recolección de datos, sin embargo, para el estudio el autor considera la entrevista y la observación de mucha utilidad para el objeto de estudio. La misma se aplicará a dos (2) representantes y dos (02) estudiantes.

La entrevista, en opinión de Martínez, M. (2008) menciona, que en la investigación cualitativa, es un instrumento técnico que posee gran sintonía epistemológica con este paradigma y su metodología; por lo que aportará una estructura de base para desarrollar la temática seleccionada por el autor del presente estudio.

En cuanto a los instrumentos de registros de las entrevistas, se utilizarán grabadora y hojas de recordatorio. Todo ello con la intención de dar fidelidad a las respuestas y opiniones para una mejor utilización de los datos. 


\subsection{Credibilidad de la Información.}

La credibilidad y validez de una investigación, tal y como lo establece, Martínez (ob.cit.) tiene un alto nivel de validez si al observar, medir o apreciar una realidad se observa, mide o aprecia esa realidad y no otra, lo que conlleva a que la validez se defina como el grado o nivel en que los resultados de la investigación reflejen una imagen clara y representativa de una realidad situacional dada; constituyendo este hecho la validez interna.

A fin de establecer la credibilidad y validez de la información, se utilizará la triangulación de la información de los actores sociales involucrados en la investigación y de las técnicas manejadas. En este orden Martínez (2008) argumenta:

... la credibilidad de la información puede variar mucho: los informantes pueden mentir, omitir datos o tener una visión distorsionada de las cosas. Será necesario contrastarla con lo de los otros o recogerla en momentos diferentes: la triangulación con distintas fuentes de información y con diversos métodos puede resultar muy valiosa (pág. 120).

La credibilidad de la información que se recabe se realizara triangulando la misma, contrastando con la información de los tres entrevistados, con lo cual se pretende obtener una imagen clara y representativa de la situación dada, igualmente, de esta manera se triangulara la información obtenida de las dos técnicas a utilizar, la entrevista y el análisis documental.

\subsection{Procesamiento de Análisis de la Información.}

A los efectos de la investigación a realizarse, dado el enfoque cualitativo, se utilizará, la técnica de la triangulación, con la cual se establecen ciertas intersecciones o coincidencias a partir de las diferentes apreciaciones de los actores involucrados. En razón de complementar los señalamientos, para Derin y Kemis (ob.cit.) la triangulación se puede definir como la 
combinación de metodologías en el estudio de un mismo problema y consiste en un control cruzado entre diferentes fuentes de datos: personas, instrumentos, documentos o la combinación de éstos.

\section{Conclusiones}

Bajo esta tendencia de la investigación surgen las conclusiones, tomando como base los objetivos pertinentes al estudio.

De esta manera el primer objetivo definido diagnosticar la realidad social y técnica del ambiente escolar para la construcción de cercas vivas, con este objetivo se pretende, realizar el diagnóstico para examinar las necesidades en cuanto a la construcción de las cercas vivas. En este momento, se realiza una descripción detallada del escenario que se desea transformar mejorar, es describir lo más objetivamente posible la realidad y de allí determinar si se hace necesario proponer una alternativa para mejorar la problemática.

En cuanto al segundo objetivo, el cual hace referencia Proporcionar las herramientas teóricas y prácticas a los alumnos para la construcción de cercas vivas, se especifica en los aportes pedagógicos que se les brinde a los alumnos en relación a la importancia de las cercas vivas en la conservación ambiental.

En lo que respecta al tercer objetivo, ejecutar un plan para la construcción de cercas vivas, el mismo hace referencia a la aplicación de un plan de actividades, donde se involucre a los actores principales de la institución.

Por último, el cuarto objetivo, lograr la participación activa de la comunidad educativa en la construcción de las cercas vivas, con él se pretende involucrar a la comunidad educativa en general, partiendo de la realidad existente en la institución, como es la aplicación de un plan para la preservación del ambiente basado en la construcción de cercas vivas. 
Este objetivo de igual manera persigue, crear una conciencia conservacionista en el resguardo de los recursos naturales, en este caso la vegetación arbórea, en tal sentido crear valores basados en la educación ambiental, el cual les permita en el futuro llevar a la practica en el contexto de su diario vivir.

\section{Referencias}

Cerda, G, H. (2003). Cómo elaborar proyectos. Bogotá: Editorial Magisterio.

Constitución de la República Bolivariana de Venezuela. (2000). Gaceta Oficial de la República Venezuela no $\mathbf{3 6 8 6 0 .}$

Currículo Básico Nacional Bolivariano (2007). Edición: Fundación Centro Nacional para el Mejoramiento de la Enseñanza de la Ciencia, CENAMEC, 2007. Depósito Legal: If51620073705047.

Fidias, A, G. (2012). El proyecto de Investigación. Introducción a la metodología científica. 6ta Edición, Editorial Episteme, Caracas.

Hernández, S, R. Fernández y Baptista, C, L. (2010). Metodología de la Investigación. 4ta Edición, México: McGraw-Hill. México.

Ley Orgánica de Educación (2009). Gaceta Oficial Número 5.929 Extraordinaria del 15 de agosto de 2009. Venezuela.

Ley Orgánica del ambiente (2006). Gaceta Oficial Palacio de Miraflores, en Caracas, a los veintidós días del mes de diciembre de dos mil seis. Años $196^{\circ}$ de la Independencia y $147^{\circ}$ de la Federación.

Ley orgánica para la protección del Niño, Niña y del Adolescente (2014).

Resolución Nro 031-2014, de fecha 01 de julio de 2014, Gaceta Oficial de la República Bolivariana de Venezuela.

Ley del Plan de la Patria (2013). Segundo Plan Socialista de Desarrollo Económico y Social de la Nación 2013-2019. Publicado en Gaceta 
Oficial de la República Bolivariana de Venezuela. No 6.118 Extraordinario, de 2013.

Maldonado, H. (2011). El impacto ambiental en Venezuela. [Artículo en línea]. Recuperado de:

http://webdelprofesor.ula.ve/nucleotachira/malhec/maldo h/el impacto ambiental en venezuela.pdf

Martínez, M, M. (2008). Ciencia y arte en la metodología Cualitativa. México: Trillas.

Mujica, S. (2014). Estado de la educación ambiental venezolana a partir de experiencias exitosas. Revista Electrónica ONHISREMI, Volumen 12, Número 2-3, 2014 [Artículo en línea]. Recuperado de: http://www.rieoei.org/oeivirt/rie11a05.pdf

Ramírez, J. (2011). Informe de impacto ambiental en Venezuela. [Artículo en línea]. Recuperado de:

http://www.everde.cl/2011/06/informe-de-impacto-ambiental-en.html

Tobar, L, D. (2010). Las cercas vivas ayudan a la conservación de la diversidad de mariposas en paisajes agropecuarios. Revista de Biología Tropical Rev. biol trop vol. 58 nro 1, San José mar, 2010. [Artículo en línea]. Recuperado de:

http://www.scielo.sa.cr/pdf/rbt/v58n1/a32v58n1.pdf

Universidad Pedagógica Experimental Libertador (2014). Manual para realizar trabajos de grado y Tesis Doctorales. II Edición. Caracas.

Vitalis (2013). Situación Ambiental de Venezuela. Revista digital Editor y Compiladores: Silva M. Da, Lizarazo, A. Recuperado de: http://www.ucv.ve/fileadmin/user upload/cenamb/Situacion-Ambientalde-Venezuela-2012.pdf

Vivas, O. (2012). Implementación de un plan de reforestación en el sector el paradero. Universidad Yacambú Barquisimeto [Trabajo de grado en línea]. Recuperado de: 
https://es.slideshare.net/jyvo22/trabajo-final-jani-vivas

Yepes, A. (2012). Deforestación en américa latina es total mente grave.

[Artículo en línea]. Recuperado de:

http://www.carbonoybosques.org/ultimas noticias/deforestacion en a

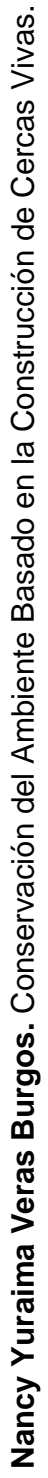
merica latina es particularmente grave.html 


\section{Nancy Yuraima Veras Burgos}

e-mail: nancyura@hotmail.es

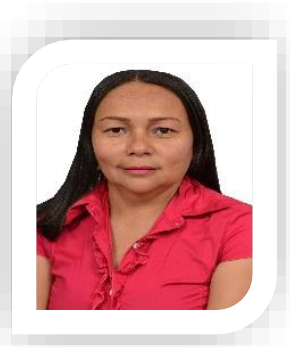

Nacida en el Tambor distrito Obispo estado Barinas, Venezuela. El 23 de Octubre de 1975, egresada de la

Universidad Pedagógica Experimental Libertado (UPEL- Barinas),con el título de Profesora en Educación Integral, cursante del Posgrado en la maestría Educación Ambiente y desarrollo (UPEL Barinas), realice cursos de secretariado comercial, en el instituto sanfrancisco de Asís, curso de Windows inicial en el instituto San Rafael , curso de primeros auxilios en la Cruz Roja , taller el hombre y su ambiente, (UNELLEZ) Barinas, trayectoria docente de 08 años de servicio como Docente de aula en las escuelas del Ner 315, escuela Estadal Costa de Morrocoy, escuela Nacional Pablo María Aliza y actualmente desempeño mis labores en la escuela Estadal Vainilla San Silvestre Barinas. 